\title{
EIF2A-dependent translational arrest protects leukemia cells from the energetic stress induced by NAMPT inhibition
}

\author{
Chiara Zucal ${ }^{1 \dagger}$, Vito G. D'Agostino ${ }^{1 \dagger}$, Antonio Casini' ${ }^{2}$, Barbara Mantelli' ${ }^{1}$, Natthakan Thongon ${ }^{1}$, Debora Soncini ${ }^{4}$, \\ Irene Caffa ${ }^{3}$, Michele $\mathrm{Cea}^{3}$, Alberto Ballestrero ${ }^{3}$, Alessandro Quattrone ${ }^{4}$, Stefano Indraccolo ${ }^{5}$, Alessio Nencioni ${ }^{3^{*}}$ \\ and Alessandro Provenzani ${ }^{1 *}$
}

\begin{abstract}
Background: Nicotinamide phosphoribosyltransferase (NAMPT), the rate-limiting enzyme in NAD ${ }^{+}$biosynthesis from nicotinamide, is one of the major factors regulating cancer cells metabolism and is considered a promising target for treating cancer. The prototypical NAMPT inhibitor FK866 effectively lowers NAD ${ }^{+}$levels in cancer cells, reducing the activity of $\mathrm{NAD}^{+}$-dependent enzymes, lowering intracellular ATP, and promoting cell death.

Results: We show that FK866 induces a translational arrest in leukemia cells through inhibition of MTOR/4EBP1 signaling and of the initiation factors EIF4E and EIF2A. Specifically, treatment with FK866 is shown to induce 5'AMP-activated protein kinase (AMPK) activation, which, together with EIF2A phosphorylation, is responsible for the inhibition of protein synthesis. Notably, such an effect was also observed in patients' derived primary leukemia cells including T-cell Acute Lymphoblastic Leukemia. Jurkat cells in which AMPK or LKB1 expression was silenced or in which a non-phosphorylatable EIF2A mutant was ectopically expressed showed enhanced sensitivity to the NAMPT inhibitor, confirming a key role for the LKB1-AMPK-EIF2A axis in cell fate determination in response to energetic stress via $\mathrm{NAD}^{+}$depletion.

Conclusions: We identified EIF2A phosphorylation as a novel early molecular event occurring in response to NAMPT inhibition and mediating protein synthesis arrest. In addition, our data suggest that tumors exhibiting an impaired LBK1- AMPK- EIF2A response may be especially susceptible to NAMPT inhibitors and thus become an elective indication for this type of agents.
\end{abstract}

Keywords: NAMPT, EIF2A, AMPK, Energetic stress, Translation arrest, UPR

\section{Background}

Aberrant activation of metabolic pathways has emerged as an hallmark of proliferating cancer cells and pharmaceutical approaches targeting cell metabolism hold potential for treating cancer [1]. Nicotinamide adenine dinucleotide $\left(\mathrm{NAD}^{+}\right)$plays a key role in different biochemical processes, acting as a coenzyme in redox reactions or as a substrate for $\mathrm{NAD}^{+}$degrading enzymes, such as poly(ADP-ribose) polymerases (PARPs), cluster of differentiation 38 (CD38), and sirtuins. Intracellular $\mathrm{NAD}^{+}$is continuously

\footnotetext{
* Correspondence: alessio.nencioni@unige.it; alessandro.provenzani@unitn.it ${ }^{\dagger}$ Equal contributors

${ }^{3}$ Department of Internal Medicine, University of Genoa, Genoa, Italy

'Laboratory of Genomic Screening, CIBIO, University of Trento, Trento, Italy

Full list of author information is available at the end of the article
}

replenished utilizing either tryptophan, nicotinamide, nicotinic acid or nicotinamide riboside as a substrate [2], and nicotinamide phosphoribosyltransferase, NAMPT, is the rate-limiting enzyme for $\mathrm{NAD}^{+}$biosynthesis from nicotinamide in mammalian cells [3]. High NAMPT levels, whose activity appears to be also important in the differentiation of myeloid cells [4], were shown to be required to support cancer cell growth, survival and epithelial-mesenchymal transition (EMT) transition $[5,6]$, and have been reported in different types of tumors $[7,8]$. In line with these notions, several studies have highlighted a strong activity of NAMPT inhibitors in preclinical models of inflammatory and malignant disorders, including leukemia [2, 9-11]. FK866, a prototypical NAMPT inhibitor, was found to 
promote cell death in both lymphoid- and myeloid-derived hematological malignancies and its activity clearly resulted from intracellular $\mathrm{NAD}^{+}$depletion [12-14]. Notably, opposite to cancer cells, activated immune cells [10], along with many other types of healthy cells, such as hematopoietic stem cells [12], appear unaffected by NAMPT inhibitors, and consistently, agents such as FK866 or CHS-828 are well tolerated in patients $[15,16]$.

The molecular consequences upon NAMPT inhibition are only partially understood. The induced $\mathrm{NAD}^{+}$depletion clearly affects intracellular ATP levels resulting in mitochondrial dysfunction and activation of cell death pathways: reactive oxygen species generation and activation of the apoptotic cascade have both been involved in cell demise in response to NAMPT inhibitors [17]. ATP depletion has been related to the loss of plasma membrane homeostasis invariably leading to oncosis cell death [18]. Different groups have suggested a role for autophagic cell death in the cytotoxic activity of these drugs [10, 12, 13, 19]. In particular, Cea and colleagues proposed that FK866 would induce autophagy via activation of transcription factor EB (TFEB), a master regulator of the lysosomal-autophagic pathway [20], and through MTORC1/AKT and ERK1/2 pathway inhibition [21]. There is also evidence that AMP-activated protein kinase (AMPK), an important coordinator of metabolic pathways in response to energetic fluctuations [22], is activated by FK866 in prostate cancer cells affecting lipogenesis [23] and in hepatocarcinoma cells with impact on MTOR/ 4EBP1 signaling [24]. Moreover, NAMPT-dependent AMPK activation associated with deacetylation of liver kinase B1 (LKB1), an upstream kinase of AMPK, has been linked with modulation of NAD levels and with significant impact on neuron cell survival [25]. Translation inhibition is often observed during cell stress [26] and this event often involves a re-programming of translation leading to differential regulation of mRNAs, occurring also via alternative mechanisms, aimed at reorganizing cell physiology to respond to the insult.

In this study, we focused on the pre-toxic molecular events induced by FK866 in acute lymphoblastic leukemia cells, known to be sensitive to the drug [10], in order to define the molecular mechanism favoring cell death or cell survival. A marked global protein synthesis inhibition represented an early cellular response associated with the FK866-induced energetic stress and here we show that AMPK-EIF2A is a central hub in mediating this effect and is responsible for cell fate decisions.

\section{Methods}

\section{Cell lines, primary B-CLL cell and T-ALL PDX isolation}

Human Jurkat T-cell acute lymphoblastic leukemia (TALL) cells were purchased from the InterLab Cell Line Collection bank (ICLC HTL01002). SUP-T1 cells were purchased from ATCC (CRL-1942) and Molt-4 Clone 8 from NIH AIDS Reagent Program (Catalog \#: 175). Human lung carcinoma A594 (CCL-185) and H460 (HTB-177) cells were purchased from ATCC. These cells were transduced with retroviral vectors encoding either LKB1 cDNA (pBABE-LKB1) or the pBABE control vector. Cell lines were grown in complete RPMI 1640 (Gibco Life Technologies) supplemented with $10 \%$ fetal bovine serum (FBS, Lonza), $2 \mathrm{mM}$ L-glutamine, $100 \mathrm{U} /$ $\mathrm{ml}$ penicillin-streptomycin (Lonza). All cell lines were grown at $37{ }^{\circ} \mathrm{C}$ under $5 \% \mathrm{CO}_{2}$ and regularly tested for mycoplasma contamination. For primary B-CLL cell isolation, a $5 \mathrm{ml}$ blood sample was obtained from patients presenting with marked lymphocytosis $(>20000 / \mu \mathrm{l})$ according to a protocol that was approved by the Ethics Committee of the Hospital IRCCS AOU San Martino IST in Genoa (\#840, February 18th 2011). Patients' written informed consent was collected. B-CLL cells were isolated by density gradient centrifugation on FicollHypaque (Biotest). The phenotype of the obtained cell preparations was confirmed by immunostaining with anti-CD19, anti-CD5, and anti-CD23 (Immunotech), and subsequent flow cytometric analysis. T-ALL xenografts (PD T-ALL) were established from BM (bone marrow) of newly diagnosed ALL pediatric patients, according to a protocol approved by the ethics committee of the University of Padova (Project number 16B/2013). The PD T-ALL cells used in this study have been published elsewhere [27]. At time of PD T-ALL establishment, written informed consent was obtained from the parents of the children. In vitro studies were performed with T-ALL cultures established from the spleen of the xenografts. Purity of the cultures (in terms of percentage of human CD5+ cells) was checked by flow cytometry and was always $>85 \%$. Research carried out on human material was in compliance with the Helsinki Declaration.

\section{Chemicals}

FK866 (sc-205325) was bought from Santa Cruz, Compound C (P5499), Nicotinic acid (N0761), Actinomycin D (A9415), (S)-(+)-Camptothecin (C9911), Cycloheximide (C1988), MG-132 (M7449), Doxorubicin hydrochloride (D1515) and Dexamethasone (D4902) were bought from Sigma-Aldrich, CHS-828 (200484-11-3) from Cayman chemical, Torin 1 (S2827) and Rapamycin (S1039) from Selleck Chemicals, Cisplatin (ALX-400040) from Enzo Life Sciences and Propidium Iodide Staining Solution from BD Pharmingen. Jurkat cells were treated with drugs dissolved in DMSO at the same cell density $\left(5 \times 10^{5}\right.$ cells $\left./ \mathrm{ml}\right)$.

\section{Viability assays}

Cell viability was assessed with the Annexin V-FITC Apoptosis Detection Kit I and 7-Aminoactinomycin D 
(7-AAD) Staining Solution (BD Pharmingen) according to manufacturer's instruction. $\mathrm{EC}_{50}$ values of FK866 were determined by nonlinear regression analysis (GraphPad Prism software v5.01,) vs viable cells in mock conditions (DMSO).

Jurkat, A549 and H460 cell lines were grown and treated in 96 well-plate for $48 \mathrm{~h}$. Cells were then assayed for viability using Thiazolyl blue tetrazolium bromide (MTT) M5655 (Sigma). In brief, MTT $(5 \mathrm{mg} / \mathrm{ml})$ at $10 \%$ volume of culture media was added to each well and cells were further incubated for $2 \mathrm{~h}$ at $37^{\circ} \mathrm{C}$. Then $100 \mu \mathrm{l}$ of DMSO was used to dissolve formazan. Absorbance was then determined at $565 \mathrm{~nm}$ by microplate reader. Cell survival was calculated and $\mathrm{EC}_{50}$ values were determined.

\section{Determination of NAD ${ }^{+}$-NADH and ATP levels and caspase/protease activity}

Intracellular $\mathrm{NAD}^{+}-\mathrm{NADH}$ content was assessed with a $\mathrm{NAD}^{+}$-NADH Quantification Kit (BioVision) according to the manufacturer's protocol. Intracellular ATP content was determined using Cell titer Glo Luminescent Cell Viability Assay (Promega). NAD ${ }^{+}-\mathrm{NADH}$ and ATP values were normalized to the number of viable cells as determined using Trypan Blue (Lonza). EnzChek Protease Assay Kit, containing a casein derivative labeled with green-fluorescent BODIPY FL (Life Technologies), was used to determine protease activity after treatment of $2 \times 10^{6}$ cells. Cells were washed once with PBS and lysed in $500 \mu \mathrm{l}$ of $1 \mathrm{X}$ digestion buffer, sonicated and centrifuged for $5 \mathrm{~min}$ at maximum speed. One $\mu \mathrm{l}$ of the BODIPY casein $100 \mathrm{X}$ was added to $100 \mu \mathrm{l}$ of the supernatant and incubated for $1 \mathrm{~h}$ protected from light. Fluorescence was measured and normalized to protein concentration in the cell lysates (Bradford Reagent, Sigma). Caspase-Glo 3/7 Assay (Promega) was used to quantify caspase activity.

\section{RNA and protein click-iT labeling kits}

Click-iT RNA Alexa Fluor 488 Imaging Kit (Life Technologies) was used to quantify the level of global RNA synthesis by flow-cytometry. Jurkat cells ( $3 \times 10^{6} /$ sample) were treated for $45 \mathrm{~h}$ with FK866 (or DMSO) and then incubated for $3 \mathrm{~h}$ with $1 \mathrm{X}$ EU working solution without removing the drug-containing media. EU detection was performed following the manufacturer's protocol after cell fixation and permeabilization. Click-iT AHA Alexa Fluor 488 Protein Synthesis Assay (Life Technologies) was used to measure the rate of translation. Cells ( $3 \times 10^{6} /$ sample) were treated for $45 \mathrm{~h}$ with FK866, centrifuged and incubated for $3 \mathrm{~h}$ with $50 \mu \mathrm{M}$ AHA in Lmethionine-free medium (RPMI Medium 1640, Sigma-Aldrich) containing the drug (or DMSO). After fixation and permeabilization, AHA incorporation was assessed by flow cytometry. 7-AAD Staining Solution $(0.25 \mu \mathrm{g} / \mathrm{sample})$ allowed the exclusion of non-viable cells.

\section{Western blotting, antibody list and plasmids}

Cells were lysed for 5 min on ice in RIPA lysis buffer supplemented with Protease Inhibitor Cocktail (SigmaAldrich). After sonication and clarification, equal amounts of proteins were separated by SDS-PAGE and blotted onto PVDF membranes (Immobilon-P, Millipore), as in [28]. The antibodies used were: 4EBP1 (sc-6936), p-4EBP1 (Ser 65/Thr 70; sc-12884), EIF4E (sc-9976), p-EIF4E (Ser 209; sc-12885), AKT1/2/3 (sc-8312), p-AKT1/2/3 (Ser 473; sc-7985), MTOR (sc-8319), BCL-2 (sc-509), NAMPT (sc-130058) from Santa Cruz; EIF2S1 (ab26197), p-EIF2S1 (Ser 51; ab32157), and p-MTOR (Ser 2448; ab1093) from

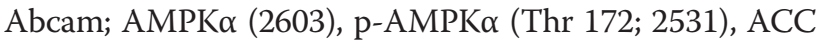
(3676) and p-ACC (Ser 79; 3661) and MCL1 (4572) from Cell Signaling. A mouse anti- $\beta$-actin antibody (3700, Cell Signaling) was used as a protein loading control. eIF2a 1 (Addgene plasmid \# 21807), eIF2a 2 (Addgene plasmid \# 21808) and eIF2a 3 (Addgene plasmid \# 21809) were a gift from David Ron. A549 cells were transfected using Lipofectamine 3000 Reagent from Life Technologies. Cells were plated in 6 well and transfected at $70 \%$ confluence for $24 \mathrm{~h}$ with $1 \mu \mathrm{g}$ of DNA. Jurkat cells were transfected for $48 \mathrm{~h}$ with $1 \mu \mathrm{g}$ of DNA in 24- well plate.

\section{Real-time PCR}

Total RNA was extracted with Quick-RNA MiniPrep kit (Zymo Research) and treated with DNAse. cDNA was synthesized using RevertAid First Strand cDNA Synthesis Kit (Fermentas) following the manufacturer's recommendation. Real-time PCR reactions were performed using the KAPA SYBR FAST Universal qPCR Kit on a CFX96 Real-Time PCR Detection System (BioRad). Relative mRNA quantification was obtained with the $\triangle \mathrm{Cq}$ method using $\beta$-actin $(A C T B)$ as housekeeping gene. Primers' sequences are reported as follows: BiP/Grp78 (Fw: TGTTCAACCAATTATCAGCAAACTC Rev: TTC TGCTGTATCCTCTTCACCAGT) ACTB (Fw: CTGGA ACGGTGAAGGTGACA Rev: AGGGACTTCCTGTAA CAATGCA) STK11/LKB1 (Fw: GAGCTGATGTCGGT GGGTATG Rev: CACCTTGCCGTAAGAGCCT).

\section{Lentiviral particles production and luciferase assay}

Lentiviral particles were produced using the pHR-SIN-RMyc-F, pHR-SIN-F-HCV-R and pHR-SIN-F-CrPV- $R$ transfer vectors [29], coding for reporter genes controlled by a cMyc-5'UTR, HCV or CrPV IRESes regulated translation, by co-transfection of $293 \mathrm{~T}$ cells with the packaging plasmid pCMV-deltaR8.91 and the VSV envelope-coding plasmid pMD2.G. Five thousand Jurkat cells/sample were transduced. After treatment with FK866, luciferase activity 
was measured using the Dual-Glo Luciferase Assay System (Promega) and normalized for protein concentration.

\section{Silencing with shRNAs}

The pLKO.1-based lentiviral plasmids containing AMPKa1 shRNA (TRCN0000000859), AMPKa2 shRNA (TRCN0000 002169) or NAMPT shRNA expression cassette (TRCN 0000116180) and (TRCN0000116181) were purchased from Sigma-Aldrich. Scramble shRNA (Addgene plasmid \#1864 [30]) was used as a control. Vectors were produced in $293 \mathrm{~T}$ cells by cotransfection of the different transfer vectors with the packaging plasmid pCMV-deltaR8.91 and the VSV envelope-coding plasmid pMD2.G. 1 million of Jurkat cells were transduced with lentiviral particles expressing the control (shSCR) or NAMPT-silencing short hairpin RNA (shNAMPT) by spinning them down with vector-containing supernatants for $2 \mathrm{~h}$ at $1600 \mathrm{xg}$ at room temperature and leaving them incubate overnight at $37^{\circ} \mathrm{C}$ without replacing the transduction supernatant. After changing the medium, the cells were further incubated for $72 \mathrm{~h}$ before collection for WB.

For AMPK silencing experiments, Jurkat cells were first transduced with the shRNA vector targeting the $\alpha 1$ subunit (shAMPK $\alpha 1$ ) as reported before. After $24 \mathrm{~h}$ from the first transduction the cells were then transduced again, following the same protocol, with the lentiviral vector coding for the shRNA targeting the AMPK $\alpha 2$ subunit (shAMPK $\alpha 2$ ). After changing the medium the next morning, the cells were further incubated for $48 \mathrm{~h}$ and then treated for additional $48 \mathrm{~h}$ with or without (DMSO) $5 \mathrm{nM}$ of FK866.

To obtain LKB1 silencing, pLKO.1 transfer vectors were prepared by cloning annealed oligos coding for shRNAs (clone TRCN0000000408 for LKB1-A and clone TRCN0000000409 for LKB1-B) into the TRC cloning vector (Addgene plasmid \#10878 [31] according to the TRC standard protocol. Cells were transduced by spinning them down with vector-containing supernatants and leaving them incubate overnight. After changing the medium, the cells were incubated for $72 \mathrm{~h}$ and then treated for additional $48 \mathrm{~h}$ with or without FK866.

\section{Statistical analysis}

Experiments were performed in biological triplicates. $T$ test was used to calculate final $p$-values, without assuming variances to be equal (Welch's $t$-test). $P$-value $<0.05$ was considered statistically significant.

\section{Results}

\section{Sensitivity of leukemia cells to the NAMPT inhibitor} FK866

FK866 was previously shown to have cytotoxic activity at nanomolar concentrations against different types of hematological malignancies, including myeloid and lymphoid leukemias and multiple myeloma [12, 21]. We monitored FK866-induced cell death in Jurkat cells by quantifying early and late apoptosis with 7AAD and Annexin V staining. In line with previous reports, FK866 cytotoxic activity started to become evident between 48 and $72 \mathrm{~h}$ of exposure with approximately 74 and $47 \%$ of viable cells left at these time points when cells are treated with FK866 100 nM (Fig. 1a), respectively. This suggests the existence of a lag phase through which cells can cope with the energetic shortage. Starting from a concentration of $10 \mathrm{nM}$, FK866 cytotoxic activity reached a plateau and an $\mathrm{EC}_{50}$ of $5.3 \mathrm{nM}$ could be estimated after $48 \mathrm{~h}$ of exposure (Fig. 1a). Indeed, at $120 \mathrm{~h}$ we measured an effective $\mathrm{IC}_{50}$ of $10 \mathrm{nM}$, highlighting the inability of these cells to compensate for the energetic stress induced by FK866 in long term treatment (Fig. 1a). Cell cycle analysis of FK866-treated cells, at $48 \mathrm{~h}$, showed a non-significant accumulation of cells in $\mathrm{G} 2 / \mathrm{M}$ phase, while, as predicted, serum starvation resulted in accumulation of cells in G0/G1 phase (Fig. 1b and Additional file 1A). Forty-eight hours treatment with FK866 led to approximately $25 \%$ of cell death, but did not lead to massive protease or caspase activation (Fig. 1c and d). However, 5 nM FK866 was sufficient to effectively reduce $\operatorname{NAD}^{+}(\mathrm{H})$ and ATP levels in Jurkat cells, representing a pre-toxic experimental condition to apply for further experiments (Fig. 1e).

\section{FK866 and NAMPT ablation blocks cap-dependent} translation, but not gene transcription, through MTOR/ 4EBP1, EIF4E, and EIF2A inhibition in cancer cells

We assessed the impact of FK866 on global transcriptional and translational efficiencies in Jurkat cells. Global RNA transcription and translation were monitored using the Click-it chemistry and flow-cytometry by the incorporation of the nucleoside analog 5-ethynyl uridine (EU) and of an aminoacid analog (AHA), respectively. In the viable Jurkat cell population, FK866 caused a reduction in the incorporation of $\mathrm{EU}$ in a dose-dependent manner, with 70 and $55 \%$ of transcriptionally active cells in the presence of 5 and $100 \mathrm{nM}$ FK866, respectively. Thus, despite $\mathrm{NAD}^{+}$and ATP depletion, cells treated with FK866 for $48 \mathrm{~h}$ essentially retained their ability to perform RNA transcription. By contrast, even 5 nM FK866 determined a striking reduction (up to $30 \%$ ) of the fraction of viable cells showing active protein synthesis (Fig. 2a and Additional file 1B). The utilization of bicistronic reporter assays to test the efficacy of cap or IRES (Internal Ribosome Entry Site) dependent translation confirmed that FK866 induced a strong translation arrest with a major impact on cap-dependent translation in Jurkat cells (Additional file 2). 


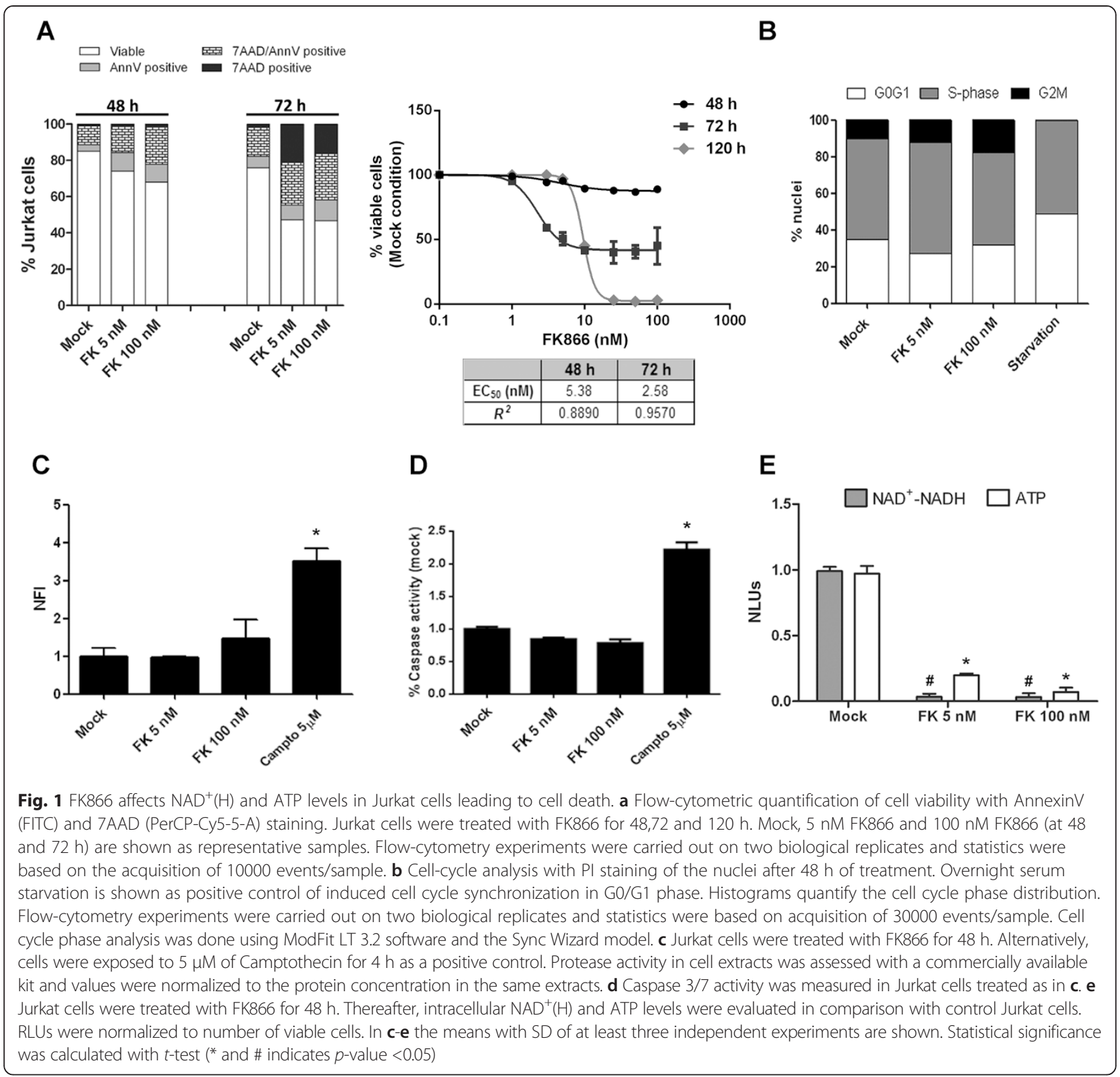

Since the initiation phase is considered the limiting step of translation [32], we evaluated the activation of three signaling pathways regulating the canonical capdependent translation process. The Mammalian Target of Rapamycin (MTOR) kinase regulates the p70 ribosomal S6 kinase (p70-S6K) and the eukaryotic translation initiation factor 4E-binding protein 4EBP1, whose phosphorylation determines EIF4E availability for its interacting partner EIF4G, which is involved in mRNA recruitment to the ribosomes for protein translation [32]. It has been recently shown that FK866 induces MTOR de-phosphorylation [24], thereby inducing autophagic cell death in multiple myeloma cells [21, 33]. As shown in Fig. 2b, Jurkat cells treated with FK866 indeed showed a marked de-phosphorylation of MTOR and 4EBP1. Enhanced AKT phosphorylation at Ser-473 was also observed (Fig. 2b), which is in line with the paradoxical activation of AKT by MTORC2 complex following inhibition of MTOR as reported with different MTOR inhibitors in multiple myeloma cells [34]. Notably, treatment with FK866 led to a previously unappreciated de-phosphorylation of EIF4E on serine 209, suggesting that the MAP Kinase Interacting Serine/ Threonine Kinase (MNK)-dependent pathway is also affected [35] (Fig. 2c), and to an increased phosphorylation on Ser-51 of EIF2A, an initiation factor that transfers methionyl-initiator tRNA (Met) to the small ribosomal subunit. When phosphorylated (Fig. 2c), EIF2A loses its 


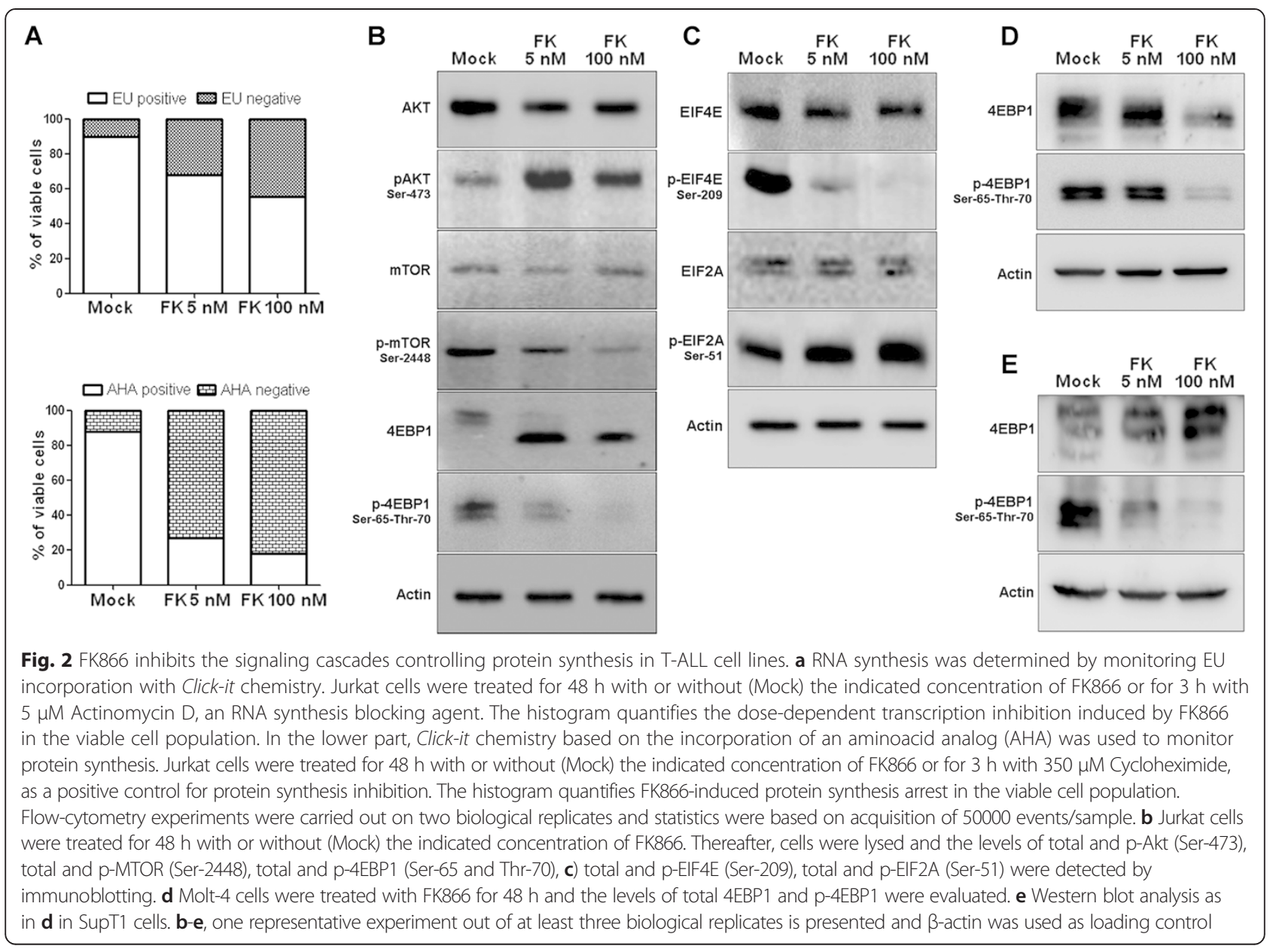

ability to exchange GDP and GTP, impairing the formation of a complex with the EIF2B subunit and thus preventing translation initiation [36]. Analogously, SUP-T1 and Molt-4 Clone 8 T-ALL cell lines presented the same FK866-induced inhibition of the activation of 4EBP1, supporting the existence of a general mechanism underlying the FK866-induced translational arrest in leukemia cells (Fig. 2d, e). These effects were also observed using another inhibitor of NAMPT enzymatic activity, CHS828 (Additional file 3A), but not with other commonly used chemotherapeutics as cisplatin, doxorubicin, dexamethasone and rapamycin, at equivalent pre-toxic doses. Indeed, FK866 induced a stronger protein synthesis arrest than the MTOR inhibitor rapamycin suggesting that this event is a molecular hallmark of FK866. Additionally, FK886 concomitantly induced EIF2A phosphorylation and 4EBP1 de-phosphorylation, uniquely among all the other drugs, thus mechanistically supporting the strong protein synthesis arrest. The other drugs tested were ineffective (Additional file 3B, C, D). In conclusion, these experiments show the modulation of several hubs of the signaling apparatus controlling translation initiation in response to FK866, providing a robust explanation for the marked protein synthesis inhibition observed after drug treatment.

\section{FK866 induces AMPK and EIF2A phosphorylation in Jurkat} and primary leukemia cells

In view of the strong translation inhibition and considering its energy-sensing activity in controlling translation [37], we investigated in Jurkat cells the impact of FK866 and CHS-828 on the phosphorylation status of AMPK, whose activation has been previously shown to be induced by FK866 in prostate and hepatic cancer cells [23, 24]. FK866 caused a partial reduction in total AMPK levels at the highest dose used, but, at a same time, a parallel dosedependent increase of the phosphorylation of its Thr-172 and of its bona fide target ACC (Acetyl-CoA Carboxylase) (Fig. 3a), indicating a significant activation of AMPK. We evaluated the effect of FK866 on two important antiapoptotic factors, MCL1 (Myeloid Cell Leukemia 1) and BCL-2 (B-Cell Lymphoma 2). BCL-2 protein levels were essentially not affected by FK866 treatment as compared to the strong down-regulation of MCL1 (Fig. 3a). Notably, nicotinic acid (NA) supplementation, which blocks FK866 cytotoxic activity by allowing $\mathrm{NAD}^{+}$biosynthesis through 


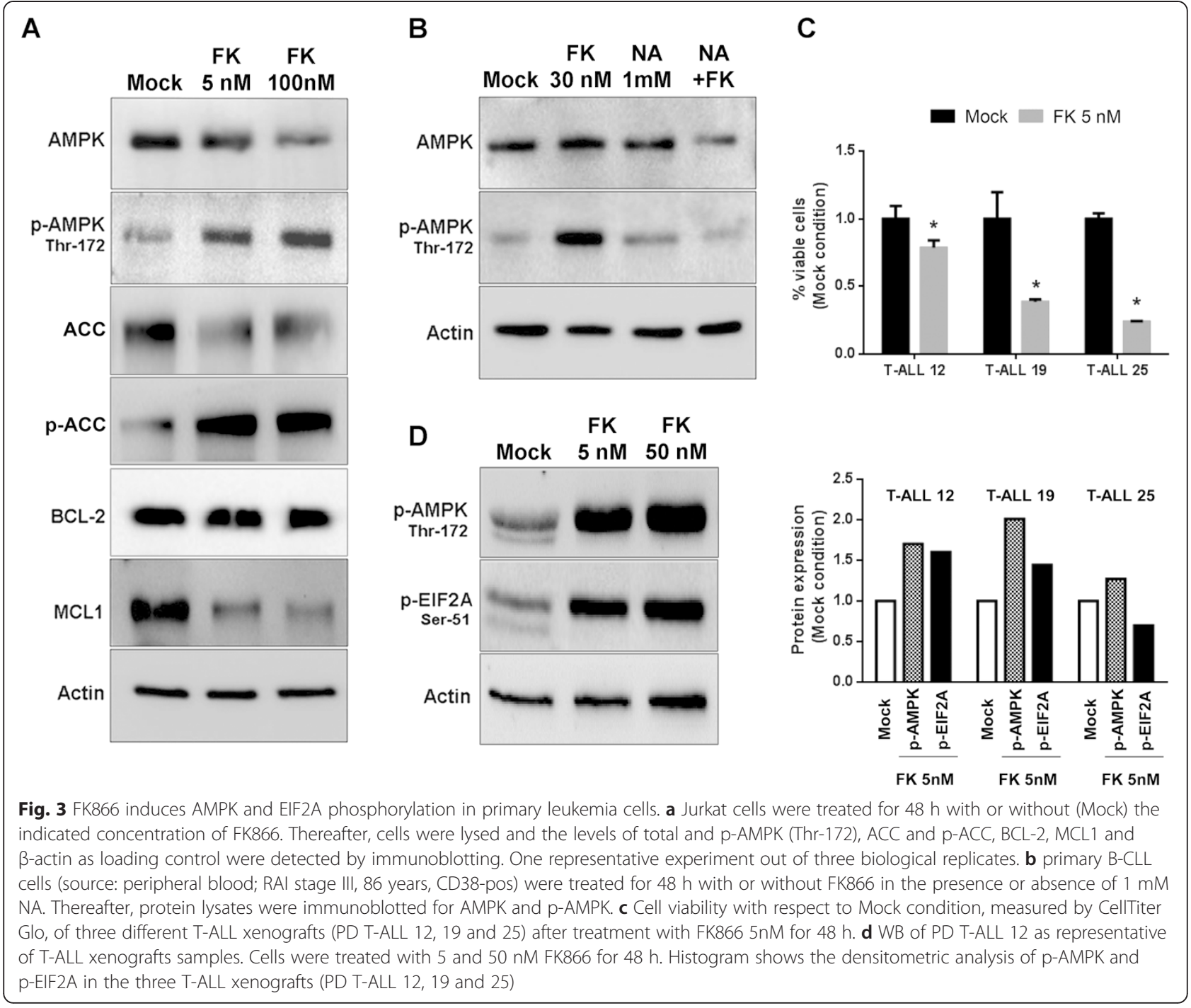

an alternative pathway (via nicotinic acid phosphoribosyltransferase, NAPRT1), completely prevented AMPK phosphorylation in primary B-CLL (Fig. 3b, Additional file 3E), confirming that $\mathrm{NAD}^{+}$depletion is responsible for AMPK activation. In patient-derived T-ALL xenografts (PD TALL) the drug induced cell death and activated AMPK as well as EIF2A phosphorylation (Fig. 3c and d), demonstrating that this molecular event is not limited to cell line models but is also present in primary leukemia cells.

\section{EIF2A phosphorylation precedes 4EBP1 de- phosphorylation in Jurkat cells}

NAMPT expression level during FK866 treatment remained unchanged as expected (Fig. 4a). Genetic ablation of NAMPT by lentiviral transduction in Jurkat cells (Fig. 4b) lowered $\operatorname{NAD}^{+}(\mathrm{H})$ level to $75 \%$ of the control while ATP level was not significantly decreased thus inducing an intermediate condition of energetic stress compared to the one obtained with 5 nM FK866 administration (Fig. 4c). In these conditions of mild stress, AMPK was marginally activated but, nevertheless, we observed a significant phosphorylation of EIF2A but not the de-phosphorylation of 4EBP1, suggesting that the first event precedes the second one (Fig. 4d). Importantly, we observed a clear down-regulation of MCL1, as observed with FK866 treatment (Fig. 3a), suggesting that EIF2A activation is an early response to $\operatorname{NAD}^{+}(\mathrm{H})$ shortage.

\section{FK866-induced AMPK activation regulates EIF2A phosphorylation}

To formally assess the role of AMPK in FK866-induced translational arrest, we pharmacologically blocked AMPK with Compound $\mathrm{C}$, a small molecule inhibitor of this enzyme, although not selective [38]. In addition, we down-regulated AMPK using lentiviral transduction of shRNAs. Compound $\mathrm{C}$ administration to Jurkat cells treated with FK866 abrogated AMPK phosphorylation, 


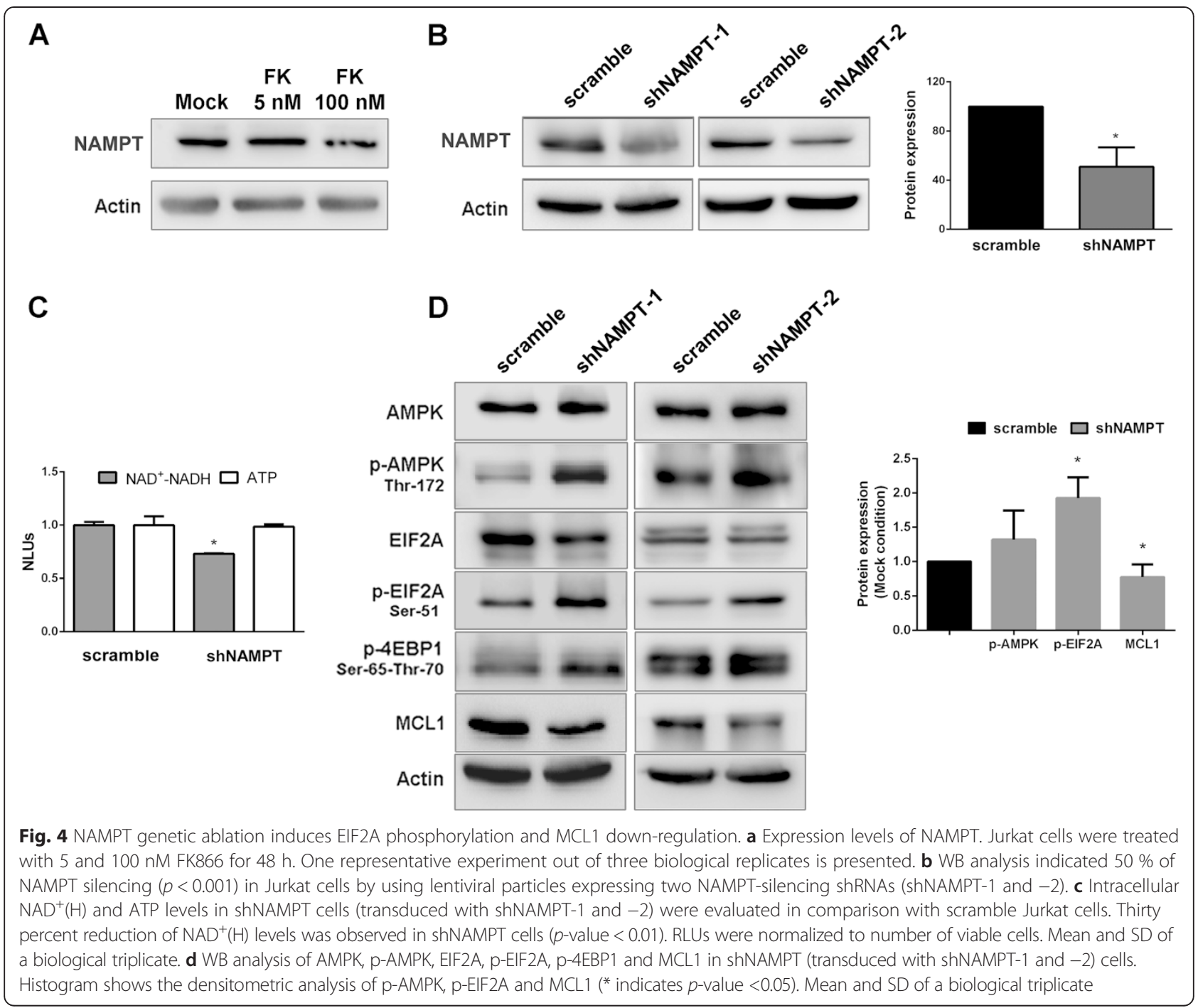

reactivated the MTOR/4EBP1 pathway and restored EIF2A in its un-phosphorylated state (Fig. 5a). Rescue experiments with Compound $\mathrm{C}$ did not show any downregulation of MCL1 protein level with no change in BCL2 expression (Fig. 5a). Co-treatment with Compound C partially reverted FK866-induced ATP loss but activated the apoptotic response (Fig. 5b). Downregulation of AMPK was achieved by targeting both AMPK $\alpha 1$ and AMPK $\alpha 2$ isoforms (shAMPK cells). We then exposed silenced and control (scramble) cells to 5 nM of FK866 for $48 \mathrm{~h}$ (Fig. 5c). In shAMPK cells we observed a significant decrease of EIF2A phosphorylation but not of 4EBP1 de-phosphorylation. This supports the notion that FK866-induced AMPK activation is primarily involved in the regulation of EIF2A phosphorylation and subsequently in 4EBP1 de-phosphorylation (Fig. 5c). Importantly, shAMPK Jurkat cells showed an increased sensitivity to FK866 with respect to control cells, as revealed by PI staining and flow-cytometry (Fig. 5d), pointing out the protective effect of AMPK in FK866induced stress conditions.

\section{EIF2A mediates the AMPK pro-survival effect during FK866 treatment}

Given the protective role of AMPK in a context of FK866-sensitive cancer cell, we hypothesized that the liver kinase B1 (LKB1), a well-established AMPK regulator, can also exert the same protective effect. Indeed, genetic ablation of LKB1 in Jurkat cells led to an increase toxicity of FK866 treatment (Fig. 6a). Accordingly, we used two lung adenocarcinoma cell lines (H460 and A549), bearing genetic inactivation of LKB1 to prove the dependency of FK866 efficacy on the activation of the LKB1/AMPK pathway. These results provide a rationale for the utilization of NAMPT inhibitors in cancers with this type of genetic background. The cells were stably transduced with retroviral vectors encoding parental LKB1 cDNA (LKB1 WT) or with a control vector 


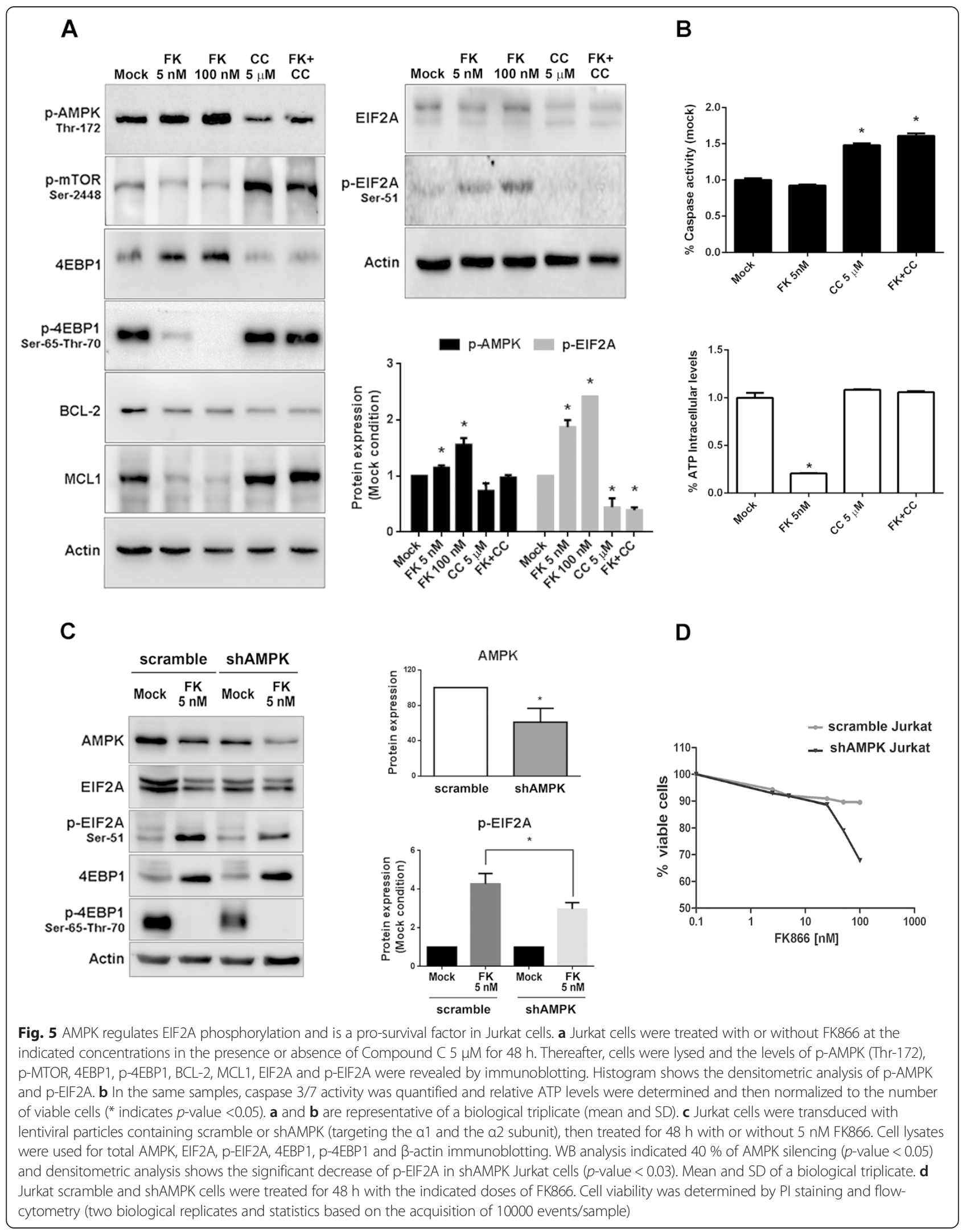




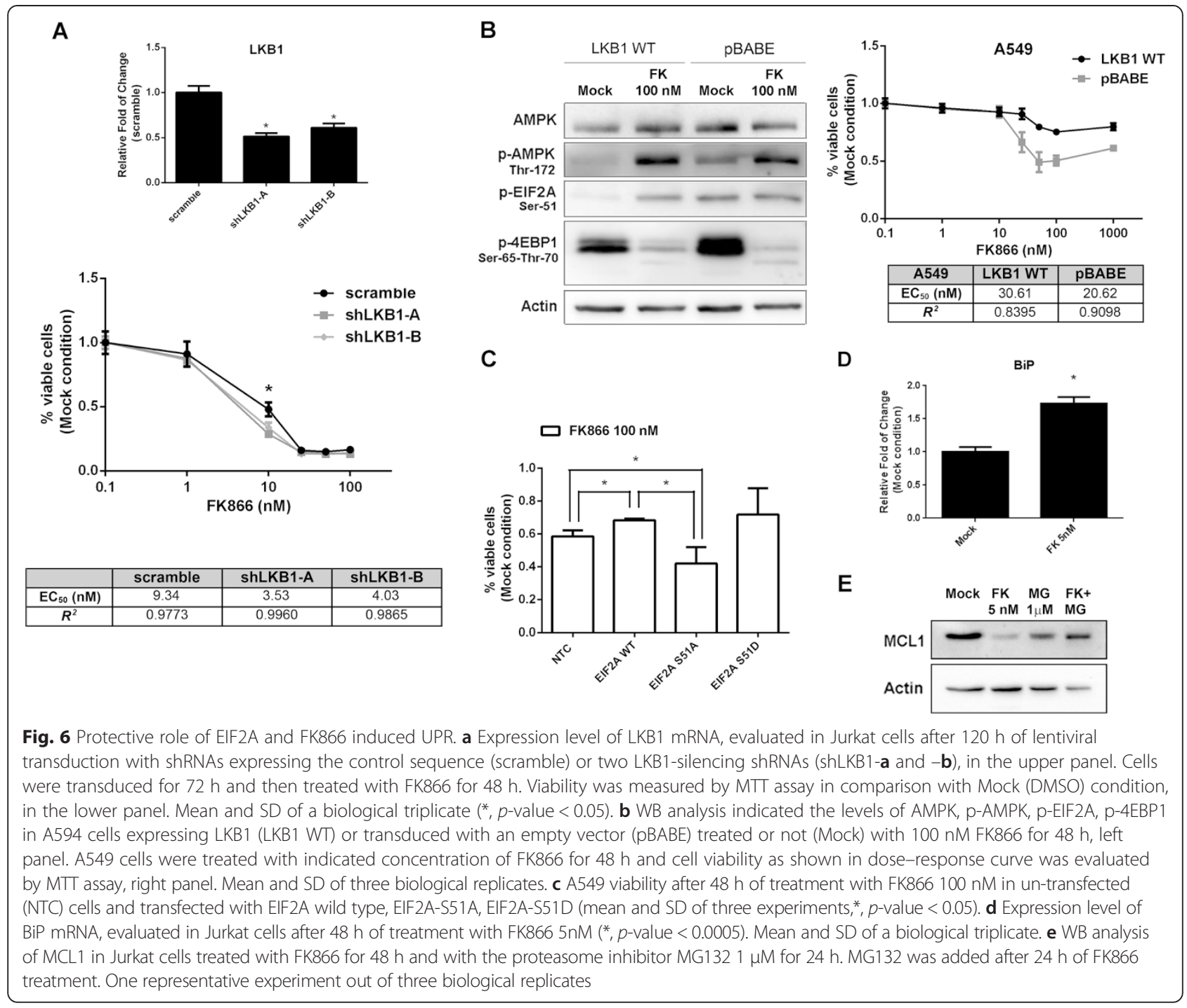

(pBABE). FK866 treatment induced AMPK and EIF2A phosphorylation in addition to 4EBP1 de-phosphorylation only when LKB1 was active. On the other hand, albeit to a different extent among the two cell lines, FK866 was not able to activate AMPK or EIF2A but was still effective in de-phosphorylating 4EBP1 when LKB1 was inactive. Viability assays indicated an increased sensitivity of LKB1 negative, EIF2A un-phosphorylated cells to FK866 compared to LKB1 expressing cells (Fig. 6b and Additional file $4 \mathrm{~A})$. Finally, in order to assess the relevance of EIF2A in mediating the AMPK induced protection from FK866, we treated with the drug A549 and Jurkat cells transfected with EIF2A, its phosphomimetic mutant S51D or with the non-phosphorylatable mutant S51A. In the context of inactive LKB1, the overexpression of EIF2A and the EIF2AS51D isoform led to a protective effect, while the alanine mutant induced an increase of cell toxicity (Fig. 6c). The same trend was observed also in Jurkat cells (Additional file 4B). Therefore, these data indicate that the translation arrest induced by EIF2A mediates the protective effect of AMPK from FK866 induced stress.

\section{EIF2A balances pro-survival and pro-death pathways}

EIF2A is a key factor regulating the translation machinery in response to a myriad of factors including nutrient depletion, presence of exogenous mRNA and the Unfolded Protein Response (UPR) following the induction of Endoplasmic Reticulum (ER) stress [39]. Indeed, FK866 induced the overexpression of BiP/Grp78 mRNA, coding for a chaperone involved in the folding of ER proteins (Fig. 6d), thus indicating UPR activation in Jurkat cells after $48 \mathrm{~h}$ treatment. Moreover, FK866 induced MCL1 down-regulation was dependent on proteasome activation as demonstrated by the rescue of its expression level by MG132 treatment in Jurkat cells (Fig. 6e) [40]. In conclusion, FK866 induces an AMPK- 
EIF2A mediated translational arrest, which is responsible for MCL1 down-regulation and the activation of the UPR response, that is a strategic pausing step necessary to protect cells from FK866-induced energetic stress.

\section{Discussion}

We investigated the link between $\operatorname{NAD}^{+}(\mathrm{H})$ depletion and cell death using a T-ALL cell model after induction of the primary effects of NAMPT inhibition, namely $\operatorname{NAD}^{+}(\mathrm{H})$ and ATP depletion, while nearly preserving total cell viability. The functional consequences of $\mathrm{NAD}^{+}(\mathrm{H})$ depletion upon FK866 treatment resulted in a marked inhibition of the three major pathways regulating the translation process and in a striking arrest of protein synthesis. Interestingly, FK866 efficacy in blocking protein synthesis was higher than all the other chemotherapeutics tested and even higher than the MTOR inhibitor rapamycin, suggesting that this is a crucial event in the cell response to FK866. This phenomenon is general because it was observed in primary leukemic samples, coming both from B-CLL and T-ALL patients, and in T-ALL derived cancer cell lines. Nicotinic acid rescue experiments, treatment with the FK866 analog CHS828 and NAMPT genetic ablation showed that translation arrest was dependent on the shortage of ATP and $\operatorname{NAD}^{+}(\mathrm{H})$ induced by the inhibition of the NAMPT catalytic function and not by unspecific FK866 effects. FK866 has been shown to have contrasting effects on AMPK. In neuronal cells FK866 decreased AMPK activation and was detrimental for neuronal survival [25], however in cancer cells that have a dysregulated metabolic demand, it has been observed the opposite. In prostate cancer cells, FK866 treatment reduced fatty acid and phospholipid synthesis, partly via AMPK activation [23]. FK866 induced activation of AMPK and subsequent decreased phosphorylation of $4 \mathrm{EBP} 1$ by MTOR has been observed in hepatocarcinoma cells. Given the importance of MTOR in sustaining cancer cell growth, this event was proposed as an effective mechanism to target cancer cells [23]. By evaluating the early molecular effects of FK866 treatment on protein synthesis, we observed the involvement of the same pathway but, in addition, we showed a protective role for AMPK and EIF2A. In our experimental conditions, the inactivation of MTOR by AMPK and consequent protein synthesis arrest had a protective effect conferring temporary resistance to the FK866-induced energetic stress. Additionally, we determined that AMPK-induced hyper-phosphorylation of EIF2A is regulated by the fluctuations of NAD availability at the intracellular level. This molecular mechanism, leading to inhibition of translation initiation, followed AMPK activation. In fact, genetic AMPK down-regulation of both isoforms of the $\alpha$ catalytic subunit rescued the FK866 induced hyper-phosphorylation of EIF2A. As a further confirmation, the same results were obtained by AMPK functional ablation using the inhibitor Compound $\mathrm{C}$ or by inactivation of its upstream regulator LKB1. Indeed, rescue of 4EBP1 phosphorylation levels was observed only after Compound $\mathrm{C}$ administration, suggesting that EIF2A is a preferential target of the AMPK signaling cascade, at least in the initial phase of cell response to FK866. Interestingly, in our cell model and at the doses we used, we did not observe Compound C-induced phosphorylation of EIF2A as recently reported in different cancer cells [41]. Our cell model resembles the AICAR-induced AMPK activation that leads to EIF2A phosphorylation in adipocytes, an event shown to be crucial for AMPK-induced apoptosis [42], and supports the idea that FK866 induced activation of the AMPK-EIF2A axis can be a novel pathway to be investigated to elucidate the pharmacology of FK866.

Many types of cancer, as sporadic lung, cervical, and endometrial cancers, carry LKB1 deficiency that can be exploited with metabolic drugs since these cells are unable to appropriately respond to metabolic stress [43]. Given the protective role of LKB1/AMPK pathway against FK866, our study suggests the utilization of FK866 as a metabolism-based cancer therapeutic to selectively target LKB1-deficient tumors. Indeed in cells lacking a functional LKB1 pathway, metabolic stress has been demonstrated to result in rapid apoptosis as the cells are unable to sense energetic stresses and activate mechanisms to restore energy homeostasis [44].

Previous studies have shown that inhibition of the MTOR/4EBP1 pathway in leukemia cells leads to a reduction in the levels of the anti-apoptotic protein MCL1, with important implications for chemosensitivity [45]. Down-regulation of MCL1 through inhibition of translation has been clearly associated with enhanced lethality in Jurkat cells [46]. Importantly, FK866 administration led to smooth death [40] via EIF2A-dependent MCL1 down-regulation consequent to translation arrest and simultaneous proteasome activation. Indeed, MCL1 intracellular levels were shown to be strictly dependent on the activation of EIF2A [47] and AMPK [48], and to the subsequent translation arrest. This could provide a molecular explanation for the anti-leukemic activity of NAMPT inhibitors. Notably, the ectopic expression of the non-phosphorylatable mutant EIF2A-S51A increased FK866 toxicity. Therefore the activation of the AMPKEIF2A axis is essential for the tumor cell to adapt to the shortage of $\mathrm{NAD}^{+}(\mathrm{H})$. For example, the increased expression level of BiP mRNA is a specific adaptive response observed in the integrated stress response (ISR) and translational repression [49]. The exacerbation of proteasome inhibition with bortezomib has been shown to potentiate FK866 efficacy through the activation of 
the caspases' cascade [40]. Here we show the relevance of EIF2A activation in this mechanism. Additionally, the synergistic effect of FK866 with cyclosporine in leukemia cells has been ascribed to the activation of the UPR [50]. This suggests that the exacerbation of the UPR, which is dependent on EIF2A, can be thought as a relevant strategy to potentiate the effect of FK866 in conditions in which activation of the EIF2A-dependent UPR is desirable, i.e., diabetes, atherosclerosis, or neurodegenerative disorders [51]. Indeed, FK866 effects on translation resemble the ones induced by metformin, a well-known AMPK activator with antidiabetic and antitumoral properties [52, 53]. Finally, de-phosphorylation of EIF4E, never linked to NAMPT inhibitors or AMPK activation before, completes the general picture of a global inhibition of the translation process, even though the mechanism leading to upstream MNK activation has not been investigated yet.

\section{Conclusions}

In conclusion, this work describes the activation of a complex signaling network in which the AMPK-EIF2A axis is responsible for the early cellular response to the metabolic stress produced by FK866. In an experimental condition in which catastrophic proteolytic cascades are not yet started but the energetic demand is high, EIF2A acts as an early master regulator of cell fate, blocking anabolic processes and, at the same time, modulating cell death and adaptive pathways. Therefore EIF2Adependent processes, such as protein synthesis and UPR, acquire fundamental relevance in explaining the mechanism of action of NAMPT inhibitors.

\section{Additional files}

\section{Additional file 1: Cell-cycle analysis and Click-iT detection of RNA} and Protein synthesis. A) Cell-cycle analysis with PI staining of the nuclei after $48 \mathrm{~h}$ of treatment. Overnight serum starvation is shown as a positive control of induced cell cycle synchronization in G0/G1 phase. Cell phase analysis was done with ModFit LT 3.2 software by using the Sync Wizard model (30000 cells/sample in biological duplicate). B) Jurkat cells were treated for $48 \mathrm{~h}$ with or without (Mock) the indicated concentration of FK866 or for $3 \mathrm{~h}$ with $5 \mu \mathrm{M}$ Actinomycin D, an RNA synthesis blocking agent, then subjected to Click-it biochemistry and flow-cytometry analyses including 7-AAD to identify living cells. C) Jurkat cells were treated for $48 \mathrm{~h}$ with or without (Mock) the indicated concentration of FK866 or for $3 \mathrm{~h}$ with $350 \mu \mathrm{M}$ Cycloheximide, as a positive control for protein synthesis inhibition, then stained as in B. In B and C. Experiments were carried out on two biological replicates (50000 events/sample). (PDF $1562 \mathrm{~kb}$ )

Additional file 2: Luciferase assays. A) Light units, normalized to protein concentration, of RLuc-cMyc 5'UTR IRES-FLuc reporter vector transduced in Jurkat cells with lentiviral particles after $48 \mathrm{~h}$ of treatment with or without (Mock) the indicated concentration of FK866. Two hour treatment with $250 \mathrm{nM}$ of Torin 1 served as a positive control for IRESdependent protein translation ( $p$-value $<0.05$ ). B) Light units, normalized to protein concentration, of FLuc-HCV-RLuc and FLuc-CrPV-RLuc reporter vectors transduced in Jurkat cells with lentiviral particles. Cap-dependent translation (FLuc) was strongly reduced with $5 \mathrm{nM}$ and $100 \mathrm{nM}$ FK866
(48 h) in comparison to Mock condition ( $p$-value <0.0001). RLuc signal is not shown because of its low level and its variability between technical and biological replicates. Cells transduced with the pHR-SIN-F-HCV-R were serum starved for $5 \mathrm{~h}$ as a positive control of IRES activation, as shown in the graph ( $p$-value $<0.05$ ). In A and B data are represented as mean and SD of three independent experiments. (PDF $584 \mathrm{~kb}$ )

Additional file 3: Effects of CHS-828 and chemotherapeutics on protein translation. A) Jurkat cells were treated for $48 \mathrm{~h}$ with or without (Mock) the indicated concentration of CHS-828. Caspase 3/7 activity was quantified (using $5 \mu \mathrm{M}$ of Camptothecin for $4 \mathrm{~h}$ as a positive control of apoptosis) and relative ATP levels were determined and then normalized to the number of viable cells. The levels of total AMPK, p-AMPK, total EIF2A and p-EIF2A, total 4EBP1, p-4EBP1 were evaluated by WB. Histogram shows the densitometric analysis of p-AMPK and p-EIF2A (* indicates $p$-value $<0.05)$. Mean and SD of a biological triplicate. B) Jurkat cells were treated with the indicated concentration of drugs for $48 \mathrm{~h}$ and cell viability was measured by Cell Titer Glo. Data are represented as mean and SD of three independent experiments. C) Click-it chemistry based on the incorporation of an aminoacid analog (AHA) was used to monitor protein synthesis. Jurkat cells were treated for $48 \mathrm{~h}$ with or without (Mock) the indicated concentration of FK866, Rapamycin (RAPA), Doxorubicin (DOXO), Cisplatin (CIS) and Dexamethasone (DEXA). The histogram quantifies the \% of AHA positive cells (active proteinsynthesizing cells) in the viable cell population. Flow-cytometry experiments were carried out on two biological replicates and statistics were based on acquisition of 20000 events/sample. D) Jurkat cells were treated as in C and the level of p-EIF2A and p-4EBP1 was evaluated. Histogram shows the densitometric analysis of p-EIF2A (* indicates $p$-value $<0.05$ ). Mean and SD of a biological triplicate. E) Primary B-CLL cells were treated for $48 \mathrm{~h}$ with or without 30 nM FK866 in the presence or absence of 1 mM NA. Histogram shows the densitometric analysis of p-AMPK/AMPK. (PDF $691 \mathrm{~kb}$ )

Additional file 4: Protective role of EIF2A. A) WB analysis indicated the levels of AMPK, p-AMPK, p-EIF2A, p-4EBP1 in H460 cells expressing LKB1 (LKB1 WT) or transduced with an empty vector (pBABE) treated or not (Mock) with 100 nM FK866 for 48 h, left panel. H460 cells were treated with indicated concentration of FK866 for $48 \mathrm{~h}$ and cell viability as shown in dose-response curve was evaluated by MTT assay, right panel. Mean and SD of three biological replicates. B) Jurkat viability after $48 \mathrm{~h}$ of treatment with FK866 5nM in un-transfected (NTC) cells and transfected with EIF2A wild type, EIF2A-S51A, EIF2A-S51D (mean and SD of three experiments, $\$, p$-value $<0.1$ ). (PDF $274 \mathrm{~kb}$ )

\section{Abbreviations}

4EBP1: Eukaryotic translation initiation factor 4E-binding protein 1; ACC: Acetyl-CoA carboxylase; AKT: v-akt murine thymoma viral oncogene homolog 1; AMPK: AMP-activated protein kinase; BCL-2: B-Cell lymphoma 2; B-CLL: B-cell chronic lymphocytic Leukemia; BiP: Glucose-regulated protein 78 kDa; EIF2A: Eukaryotic translation initiation factor 2A; EIF4E: Eukaryotic translation initiation factor 4E; EMT: Epithelial-mesenchymal transition; ERK: Mitogen-activated protein kinase; LKB1: Liver kinase B1; MCL1: Myeloid cell leukemia 1; MNK: MAP Kinase Interacting Serine/Threonine Kinase; MTOR: Mammalian target of rapamycin; NA: Nicotinic acid; NAD: Nicotinamide adenine dinucleotide; NAMPT: Nicotinamide phosphoribosyltransferase; T-ALL: T-cell acute lymphoblastic Leukemia; UPR: Unfolded protein response.

\section{Competing interests}

Authors declare no competing interests.

\section{Authors' contributions}

CZ and VGD carried out most of the experiments, participated in the study design and drafted the manuscript, AC cloned and produced viral vectors, $B M, N T, D S, M C$ and IC helped with immunoassays and genetic experiments, $A B, S I, A Q$ provided clinical samples and participated in the study design, AN and AP conceived the study, participated in its design and coordination and wrote the manuscript. All authors drafted, read and approved the final manuscript.

\section{Acknowledgements}

We thank Dr V. Adami, High Throughput Screening Facility, and I. Pesce, Cell Analysis and Separation Facility, University of Trento, Italy, for helpful support; 
Dr Sabatini DM for shscramble pLKO based vector provided through Addgene and Dr Root DE for pLKO.1 - TRC cloning vector. The pBABE-LKB1 vector was received from Dr. Lewis Cantley through Addgene.

\section{Financial support}

AP and AN thank the Italian Ministry of Health grant GR-2008-1135635 and FP7 project PANACREAS \#256986. AN thanks AIRC Start-Up grant \#6108, Compagnia di San Paolo, Fondazione Umberto Veronesi, Fondazione CARIGE, Università di Genova. AP thanks the CIBIO start-up grant, University of Trento.

\section{Author details}

'Laboratory of Genomic Screening, CIBIO, University of Trento, Trento, Italy. ${ }^{2}$ Laboratory of Molecular Virology, CIBIO, University of Trento, Trento, Italy. ${ }^{3}$ Department of Internal Medicine, University of Genoa, Genoa, Italy. ${ }^{4}$ Laboratory of Translational Networks, CIBIO, University of Trento, Trento, Italy. ${ }^{5}$ Istituto Oncologico Veneto IOV-IRCCS, Padova, Italy.

\section{Received: 15 April 2015 Accepted: 23 October 2015}

\section{Published online: 05 November 2015}

\section{References}

1. Kroemer G, Pouyssegur J. Tumor cell metabolism: cancer's Achilles' heel. Cancer Cell. 2008;13(6):472-82.

2. Tan B, Young DA, Lu Z-H, Wang T, Meier TI, Shepard RL, et al. Pharmacological inhibition of nicotinamide phosphoribosyltransferase (NAMPT), an enzyme essential for NAD+ biosynthesis, in human cancer cells: metabolic basis and potential clinical implications. J Biol Chem. 2013;288(5):3500-11.

3. Revollo JR, Grimm AA, Imai S. The NAD biosynthesis pathway mediated by nicotinamide phosphoribosyltransferase regulates Sir2 activity in mammalian cells. J Biol Chem. 2004:279(49):50754-63.

4. Skokowa J, Lan D, Thakur BK, Wang F, Gupta K, Cario G, et al. NAMPT is essential for the G-CSF-induced myeloid differentiation via a NAD(+)-sirtuin1-dependent pathway. Nat Med. 2009;15(2):151-8.

5. Xiao Y, Elkins K, DurieuX JK, Lee L, Oeh J, Yang LX, et al. Dependence of Tumor Cell Lines and Patient-Derived Tumors on the NAD Salvage Pathway Renders Them Sensitive to NAMPT Inhibition with GNE-618. Neoplasia. 2013;15(10):1151-60

6. Soncini D, Caffa I, Zoppoli G, Cea M, Cagnetta A, Passalacqua M, et al. Nicotinamide phosphoribosyltransferase promotes epithelial-tomesenchymal transition as a soluble factor independent of its enzymatic activity. J Biol Chem. 2014;289(49):34189-204.

7. Wang B, Hasan MK, Alvarado E, Yuan H, Wu H, Chen WY. NAMPT overexpression in prostate cancer and its contribution to tumor cell survival and stress response. Oncogene. 2011;30(8):907-21.

8. Nakajima TE, Yamada Y, Hamano T, Furuta K, Gotoda T, Katai H, et al. Adipocytokine levels in gastric cancer patients: resistin and visfatin as biomarkers of gastric cancer. J Gastroenterol. 2009:44(7):685-90.

9. Montecucco F, Cea M, Cagnetta A, Damonte P, Nahimana A, Ballestrero A, et al. Nicotinamide phosphoribosyltransferase as a target in inflammationrelated disorders. Curr Top Med Chem. 2013;13(23):2930-8.

10. Bruzzone S, Fruscione F, Morando S, Ferrando T, Poggi A, Garuti A, et al. Catastrophic NAD+ depletion in activated T Iymphocytes through Nampt inhibition reduces demyelination and disability in EAE. PLoS One. 2009:4(11):e7897.

11. Zoppoli G, Cea M, Soncini D, Fruscione F, Rudner J, Moran E, et al. Potent synergistic interaction between the Nampt inhibitor APO866 and the apoptosis activator TRAIL in human leukemia cells. Exp Hematol. 2010;38(11):979-88.

12. Nahimana A, Attinger A, Aubry D, Greaney P, Ireson C, Thougaard AV, et al. The NAD biosynthesis inhibitor APO866 has potent antitumor activity against hematologic malignancies. Blood. 2009;113(14):3276-86.

13. Nencioni A, Cea M, Montecucco F, Longo VD, Patrone F, Carella AM, et al. Autophagy in blood cancers: biological role and therapeutic implications. Haematologica. 2013;98(9):1335-43.

14. Wosikowski K, Mattern K, Schemainda I, Hasmann M, Rattel B, Löser R. WK175, a novel antitumor agent, decreases the intracellular nicotinamide adenine dinucleotide concentration and induces the apoptotic cascade in human leukemia cells. Cancer Res. 2002;62(4):1057-62.

15. Christensen MK, Erichsen KD, Olesen UH, Tjørnelund J, Fristrup P, Thougaard A, et al. Nicotinamide phosphoribosyltransferase inhibitors, design, preparation, and structure-activity relationship. J Med Chem 2013:56(22):9071-88.

16. Montecucco F, Cea M, Bauer I, Soncini D, Caffa I, Lasigliè D, et al. Nicotinamide phosphoribosyltransferase (NAMPT) inhibitors as therapeutics: rationales, controversies, clinical experience. Curr Drug Targets. 2013:14(6):637-43.

17. Cerna D, Li H, Flaherty S, Takebe N, Coleman CN, Yoo SS. Inhibition of nicotinamide phosphoribosyltransferase (NAMPT) activity by small molecule GMX1778 regulates reactive oxygen species (ROS)-mediated cytotoxicity in a p53- and nicotinic acid phosphoribosyltransferase1 (NAPRT1)-dependent manner. J Biol Chem. 2012;287(26):22408-17.

18. Del Nagro C, Xiao Y, Rangell L, Reichelt M, O'Brien T. Depletion of the Central Metabolite NAD Leads to Oncosis-mediated Cell Death. J Biol Chem. 2014;289(51):35182-92

19. Ginet V, Puyal J, Rummel C, Aubry D, Breton C, Cloux A-J, et al. A critical role of autophagy in antileukemia/lymphoma effects of AP0866, an inhibitor of NAD biosynthesis. Autophagy. 2014;10(4):603-17.

20. Settembre C, De Cegli R, Mansueto G, Saha PK, Vetrini F, Visvikis O, et al. TFEB controls cellular lipid metabolism through a starvation-induced autoregulatory loop. Nat Cell Biol. 2013;15(6):647-58.

21. Cea M, Cagnetta A, Fulciniti M, Tai Y-T, Hideshima T, Chauhan D, et al. Targeting NAD+ salvage pathway induces autophagy in multiple myeloma cells via mTORC1 and extracellular signal-regulated kinase (ERK1/2) inhibition. Blood. 2012;120(17):3519-29.

22. Hardie DG. The AMP-activated protein kinase pathway-new players upstream and downstream. J Cell Sci. 2004;117(Pt 23):5479-87.

23. Bowlby SC, Thomas MJ, D'Agostino RB, Kridel SJ. Nicotinamide phosphoribosyl transferase (Nampt) is required for de novo lipogenesis in tumor cells. PLoS One. 2012;7(6):e40195.

24. Schuster S, Penke M, Gorski T, Gebhardt R, Weiss TS, Kiess W, et al. FK866induced NAMPT inhibition activates AMPK and downregulates mTOR signaling in hepatocarcinoma cells. Biochem Biophys Res Commun. 2015;458(2):334-40.

25. Wang P, Xu T-Y, Guan Y-F, Tian W-W, Viollet B, Rui Y-C, et al. Nicotinamide phosphoribosyltransferase protects against ischemic stroke through SIRT1dependent adenosine monophosphate-activated kinase pathway. Ann Neurol. 2011;69(2):360-74.

26. Spriggs KA, Stoneley M, Bushell M, Willis AE. Re-programming of translation following cell stress allows IRES-mediated translation to predominate. Biol Cell. 2008;100(1):27-38.

27. Agnusdei V, Minuzzo S, Frasson C, Grassi A, Axelrod F, Satyal S, et al. Therapeutic antibody targeting of Notch 1 in T-acute lymphoblastic leukemia xenografts. Leukemia. 2013;28(2):278-88.

28. D'Agostino VG, Adami V, Provenzani A. A novel high throughput biochemical assay to evaluate the HuR protein-RNA complex formation. PLoS One. 2013;8(8):e72426.

29. Zufferey R, Nagy D, Mandel RJ, Naldini L, Trono D. Multiply attenuated lentiviral vector achieves efficient gene delivery in vivo. Nat Biotechnol. 1997;15(9):871-5.

30. Sarbassov DD, Guertin DA, Ali SM, Sabatini DM. Phosphorylation and regulation of Akt/PKB by the rictor-mTOR complex. Science. 2005;307(5712):1098-101.

31. Moffat J, Grueneberg DA, Yang X, Kim SY, Kloepfer AM, Hinkle G, et al. A lentiviral RNAi library for human and mouse genes applied to an arrayed viral high-content screen. Cell. 2006;124(6):1283-98.

32. Sonenberg $N$, Hinnebusch AG. Regulation of translation initiation in eukaryotes: mechanisms and biological targets. Cell Elsevier Inc. 2009;136(4):731-45.

33. Cea M, Cagnetta A, Patrone F, Nencioni A, Gobbi M, Anderson KC. Intracellular $\mathrm{NAD}(+)$ depletion induces autophagic death in multiple myeloma cells. Autophagy. 2013;9(3):410-2.

34. Shi $Y$, Yan H, Frost $P$, Gera J, Lichtenstein A. Mammalian target of rapamycin inhibitors activate the AKT kinase in multiple myeloma cells by up-regulating the insulin-like growth factor receptor/insulin receptor substrate-1/ phosphatidylinositol 3-kinase cascade. Mol Cancer Ther. 2005;4(10):1533-40.

35. Waskiewicz AJ, Flynn A, Proud CG, Cooper JA. Mitogen-activated protein kinases activate the serine/threonine kinases Mnk1 and Mnk2. EMBO J. 1997;16(8):1909-20.

36. Krishnamoorthy T, Pavitt GD, Zhang F, Dever TE, Hinnebusch AG. Tight binding of the phosphorylated alpha subunit of initiation factor 2 (elF2alpha) to the regulatory subunits of guanine nucleotide exchange 
factor elF2B is required for inhibition of translation initiation. Mol Cell Biol. 2001;21(15):5018-30.

37. Zhang C-S, Jiang B, Li M, Zhu M, Peng Y, Zhang Y-L, et al. The Lysosomal v-ATPase-Ragulator Complex Is a Common Activator for AMPK and mTORC1, Acting as a Switch between Catabolism and Anabolism. Cell Metab. 2014;20(3):526-40

38. Liu X, Chhipa RR, Nakano I, Dasgupta B. The AMPK inhibitor compound C is a potent AMPK-independent antiglioma agent. Mol Cancer Ther. 2014;13(3):596-605.

39. Wang $\mathrm{S}$, Kaufman RJ. The impact of the unfolded protein response on human disease. J Cell Biol. 2012;197(7):857-67.

40. Cagnetta A, Cea M, Calimeri T, Acharya C, Fulciniti M, Tai Y-T, et al. Intracellular $\mathrm{NAD}^{+}$depletion enhances bortezomib-induced anti-myeloma activity. Blood. 2013;122(7):1243-55.

41. Dai RY, Zhao XF, Li JJ, Chen R, Luo ZL, Yu LX, et al. Implication of transcriptional repression in compound C-induced apoptosis in cancer cells. Cell Death Dis. 2013;4:e883.

42. Dagon Y, Avraham Y, Berry EM. AMPK activation regulates apoptosis, adipogenesis, and lipolysis by elF2alpha in adipocytes. Biochem Biophys Res Commun. 2006;340(1):43-7.

43. Shackelford DB, Abt E, Gerken L, Vasquez DS, Seki A, Leblanc M, et al. LKB1 inactivation dictates therapeutic response of non-small cell lung cancer to the metabolism drug phenformin. Cancer Cell. 2013;23(2):143-58.

44. Shaw RJ, Kosmatka M, Bardeesy N, Hurley RL, Witters LA, DePinho RA, et al. The tumor suppressor LKB1 kinase directly activates AMP-activated kinase and regulates apoptosis in response to energy stress. Proc Natl Acad Sci U S A. 2004;101(10):3329-35.

45. Mills JR, Hippo Y, Robert F, Chen SMH, Malina A, Lin C-J, et al. mTORC1 promotes survival through translational control of Mcl-1. Proc Natl Acad Sci U S A. 2008;105(31):10853-8.

46. Zhou T, Li G, Cao B, Liu L, Cheng Q, Kong H, et al. Downregulation of Mcl-1 through inhibition of translation contributes to benzyl isothiocyanateinduced cell cycle arrest and apoptosis in human leukemia cells. Cell Death Dis. 2013;4:e515.

47. Fritsch RM, Schneider G, Saur D, Scheibel M, Schmid RM. Translational repression of MCL-1 couples stress-induced elF2 alpha phosphorylation to mitochondrial apoptosis initiation. J Biol Chem. 2007:282(31):22551-62

48. Pradelli LA, Bénéteau M, Chauvin C, Jacquin MA, Marchetti S, Muñoz-Pinedo C, et al. Glycolysis inhibition sensitizes tumor cells to death receptorsinduced apoptosis by AMP kinase activation leading to Mcl-1 block in translation. Oncogene. 2010;29(11):1641-52.

49. Palam LR, Baird TD, Wek RC. Phosphorylation of elF2 facilitates ribosomal bypass of an inhibitory upstream ORF to enhance CHOP translation. J Biol Chem. 2011:286(13):10939-49.

50. Cagnetta A, Caffa I, Acharya C, Soncini D, Acharya P, Adamia S, et al. APO866 Increases Antitumor Activity of Cyclosporin-A by Inducing Mitochondrial and Endoplasmic Reticulum Stress in Leukemia Cells. Clin Cancer Res. 2015:21(17):3934-45.

51. Fullwood MJ, Zhou W, Shenolikar S. Targeting phosphorylation of eukaryotic initiation factor-2a to treat human disease. Prog Mol Biol Transl Sci. 2012;106:75-106

52. Dowling RJO, Zakikhani M, Fantus IG, Pollak M, Sonenberg N. Metformin inhibits mammalian target of rapamycin-dependent translation initiation in breast cancer cells. Cancer Res. 2007;67(22):10804-12.

53. Larsson O, Morita M, Topisirovic I, Alain T, Blouin M-J, Pollak M, et al. Distinct perturbation of the translatome by the antidiabetic drug metformin. Proc Natl Acad Sci U S A. 2012:109(23):8977-82.

\section{Submit your next manuscript to BioMed Central and take full advantage of:}

- Convenient online submission

- Thorough peer review

- No space constraints or color figure charges

- Immediate publication on acceptance

- Inclusion in PubMed, CAS, Scopus and Google Scholar

- Research which is freely available for redistribution 